\title{
Bibliotecas y bibliotecarios especializados en América Latina y el Caribe
}

\author{
Sueli Angelica Do Amaral *
}

\begin{abstract}
RESUMEN
Se presentan estadísticas acerca de las condiciones sociales y económicas de algunos países de América Latina y el Caribe, condiciones muy similares entre sí. Se analiza el papel del bibliotecario especializado en el contexto de América Latina y el Caribe, con referencia a las responsabilidades del bibliotecario. Se hacen sugerencias con respecto a la participación de este tipo de profesionista en el trabajo cooperativo que se realiza en América Latina y el Caribe, y en actividades relacionadas con los movimientos de asociaciones de bibliotecarios en la región como un medio de asegurar el desempeño efectivo de estas bibliotecas especializadas de América Latina y el Caribe para satisfacer las necesidades de información del usuario.
\end{abstract}

\begin{abstract}
Similar social and economic stadistics in some countries of Latin America and the Caribbean are presented. Comments on the role of the specialized librarian in the context of Latin America and the Caribbean, with reference to the librarian's responsabilities, Suggestions are made regarding participation by this type of professional in cooperative work undertaken in Latin America and the Caribbean and in activities involving librarian association movements in the region as means for ensuring effective performance by these specialized Latin America and Caribbean libraries in providing for user information needs.
\end{abstract}

\section{LOS SERVICIOS \\ BIBLIOTECARIOS Y LOS BIBLIOTECARIOS EN AMÉRICA LATINA Y EL CARIBE}

Ce pueden encontrar muchas similitudes entre los países de América Latina y el Caribe. Las condiciones económicas, sociales, culturales y políticas, así como el desarrollo de estos países, presentan características muy parecidas, en particular con respecto a factores que ejercen gran influencia en el desarrollo del sector informativo.

En general, una gran parte de la población de estos países es pobre y analfabeta. Según el Anuario Estadístico para América Latina y el Caribe, en su edición de 1990, publicado por la Comisión Económica para América Latina y el Caribe (CEPAL), había un índice del $47 \%$ de analfabetismo entre la población mayor de 15 años en Haití, en 1990. Para ese mismo año Guatemala tenía una tasa de analfabetismo entre su población del $44 \%$, mientras que la de El Salvador era el $27 \%$. Otro cuadro estadístico en el mismo anuario brinda cifras que muestran una tendencia generalizada al indicar el porcentaje de familias que viven en la pobreza tanto en las ciudades como en todo el país. En 1967, las familias de Honduras que estaban por debajo del nivel de la pobreza era el $65 \%$ en cobertura nacional y del $40 \%$ en las familias urbanas. En 1972, Perú tenía un 50\% en todo el país y un $28 \%$ de familias urbanas que vivían en la pobreza ese mismo año. El $49 \%$ de las familias de Brasil en todo el país y el 35\% de las urbanas vivían por debajo del nivel de la pobreza. ${ }^{1}$

Además de estos problemas sociales, la deuda interna y externa de los países de
América Latina y el Caribe no resultan alentadoras. Un informe de la CEPAL de $1991^{2}$ acerca del panorama económico para América Latina estimaba que la deuda externa acumulada de la región (de 432, 000 millones de dólares estadounidenses a fines de 1990) aumentaría en términos nominales en un 3\% aproximadamente durante 1991.

Ante dificultades tan enormes, la infraestructura informativa no recibe el apoyo gubernamental necesario para desempeñar sus funciones satisfactoriamente. Aunado a ello, la falta de continuidad en la planeación gubernamental exacerba la situación. Con mucha frecuencia, las autoridades gubernamentales, si bien dan prioridad a los problemas básicos, relacionados con la educación y la salud, soslayan reconocer la importancia de la ciencia y la tecnología para el desarrollo

\footnotetext{
* Miembro de la Sección de referencias bibliográficas, Biblioteca del Senado Federal, Brasilia, Brasil
} 
de sus países. Se sabe que el número de escuelas existentes no resulta suficiente para el volumen de la población. En cuanto a las bibliotecas, la situación es más compleja, ya que no se dispone siempre de estadísticas oficiales al respecto. Por ejemplo, en Brasil no se tienen cifras oficiales sobre el número total de bibliotecas, pues el censo cultural de la educación se está elaborando.

$\mathrm{Al}$ analizar los servicios bibliotecarios en Brasil, Zamora ${ }^{3}$ enlista varios factores que tienen en común los países de la región, lo cual determina el nivel de desarrollo de la biblioteconomía de América Latina. En su estudio, la autora subraya la falta de apoyo gubernamental para la infraestructura informativa, así como la insuficiencia de los datos estadísticos oficiales sobre este sector. Además de estos factores, menciona la autora el nivel educativo de los recursos humanos que trabajan en el área, la legislación respecto a la biblioteconomía, los procedimientos para la planeación y la cooperación, la influencia ejercida por los países desarrollados, la situación del bibliotecario, las asociaciones profesionales y el impacto de la tecnología. Zamora presenta estadísticas sobre los países latinoamericanos al comentar cada uno de los factores mencionados. Para concluir pone de relieve la importancia de la actitud del bibliotecario en su relación con el usuario, la comunidad y la educación.

Aunque las observaciones de Zamora se refieren a América Latina únicamente, es importante la situación profesional del bibliotecario, independientemente de los límites geográficos de su actividad, o del tipo de biblioteca en la cual desempeña sus labores. Es fundamental la actitud del bibliotecario en el contexto del papel que tendrá la biblioteca. Saber cómo interpretar las necesidades de información de los usuarios; valorar el servicio de calidad y la interacción con la comunidad que se atiende, mostrarse receptivos alas críticas, sugerencias y quejas, valuando siempre el suministro de servicios y productos informativos, además de saber cómo establecer prioridades y resaltar los resultados; éstos son algunos de los principios básicos que deberían orientar los intereses de los bibliotecarios en cualquier cosa de biblioteca.
Independientemente del tipo de biblioteca que se considere, se debe considerar a esta organización como una "empresa", como cualquier otra, al mismo tiempo que se preserva su naturaleza específica. Es necesario reconocer a los competidores de la biblioteca dentro de su entorno específico. Si los bibliotecarios actúan en consecuencia, podrán elegir más fácilmente las oportunidades de acción y anticipar las necesidades de información de los usuarios. Lograrán entonces resultados positivos, satisfarán completamente dichas necesidades y aprovecharan mejor los recursos disponibles se encuentre la biblioteca en América Latina, el Caribe o en cualquier otro lugar del mundo.

Además, se deberá prestar atención al hecho de que el mundo está en cambio constante. Los avances tecnológicos permiten que los usuarios interesados en obtener información para emplear los medios electrónicos y tener acceso directo a la información sin la intervención de la biblioteca y sin que un bibliotecario actué como intermediario. Vivimos en una sociedad postindustrial. Y corresponde al bibliotecario acompañar esta evolución, asegurando la posición preponderante de la biblioteca como uno de los canales de acceso a la información. El hecho de que una biblioteca pueda carecer de los apoyos tecnológicos más avanzados no debiera restar interés por parte del bibliotecario para conocer la tecnología al alcance.

En este contexto, algunos estudiosos hacen proyecciones para el futuro. Algunos se muestran pesimistas y vaticinan una sociedad que prescindirá del papel impreso, por lo que no habrá lugar para la biblioteca, Nos toca creer a nosotros en un futuro en el que habrá todavía bibliotecas y bibliotecarios, a condición de que estos últimos se pan como mantenerse al tanto del progreso tecnológico. En último análisis, la que crea la innovación tecnológica es la inteligencia humana.

Entre los futurólogos, Alvin Toffler, en su análisis, denomina como la tercera ola al futuro caracterizado fundamentalmente por la importancia de la información como un insumo básico para el desarro- 1lo, Así, el papel que desempeñara el bibliotecario en este escenario cobra especial importancia, ya que el objetivo consiste en alcanzar el nivel ideal para satisfacer las necesidades de información de los usuarios en las bibliotecas de América Latina y el Caribe.

A fin de definir los parámetros del presente artículo, se abordarán sólo los aspectos relacionados con la información científica y técnica, analizando el desempeño de las bibliotecas especializadas en este contexto.

\section{PAPEL Y DESPEMPEÑO DE LAS BIBLIOTECAS ESPECIALIZADAS}

El crecimiento exponencial de los estudios científicos y técnicos en todo el mundo torna difícil el proceso de actualizarse profesionalmente. Para mantenerse al día, todo profesionista debe absorber cada año alrededor de un $20 \%$ de nuevos conocimientos, sólo para compensar la pérdida y el desgaste natural de su competencia técnica. Este fenómeno implica un impulso inevitable hacia la actualización, la cual, de programarse de manera errónea, lo conducirá a investigaciones inútiles, a una duplicación de esfuerzos y a gastos innecesarios en busca de información ya descubierta y en uso corriente.

Cada día se vuelve más necesario contar con información precisa y actualizada para llevar a cabo cualquier proyecto. Debido a ello, las organizaciones científicas y técnicas necesitan un servicio que recopile, seleccione, procese, almacene y de a conocer con gran eficiencia la información necesaria para el desarrollo de la actividad científica, de modo que la recuperación de información se realice rápidamente, y así se satisfaga la demanda de información específica por parte de cada organización.

La biblioteca especializada brinda exactamente esa clase de apoyo. Sin importar cuál sea su área de actividad, el papel que desempeñará en el contexto arriba des- 
crito merece especial atención por parte de los bibliotecarios, independientemente de su localización geográfica.

Resulta esencial cumplir una misión tan compleja e importante a fin de que se reconozca y se valore adecuadamente tanto a las bibliotecas especializadas como a los bibliotecarios mismos, ya que son ellos los profesionistas responsables de la actividad en este sector, Los bibliotecarios deberán actuar en forma dinámica, manteniéndose en contacto con el progreso tecnológico y desarrollando su potencial creador e innovador en su afán por identificar y satisfacer las demandas de información de la biblioteca especializada en que laboran.

Entre los mecanismos que han de adoptarse en este contexto es importante considerar la acción cooperativa. Ninguna corporación resulta en sí completa ni suficiente para satisfacer todas las necesidades de una organización, independientemente de lo adecuada que resulte la metodología empleada para desarrollar una colección disponible en el acervo, incluso si se cuenta con los fondos suficientes para adquirir documentos.

Este constituye sólo un ejemplo que muestra la validez del esfuerzo cooperativo. Gracias a la cooperación, varias organizaciones procuran racionalizar el uso de sus recursos, con lo que intentan contribuir de modo significativo en la búsqueda de soluciones a sus problemas en común. Los bibliotecarios debieran recurrir a esta alternativa con vistas a alcanzar un nivel de satisfacción más alto en lo que respecta a las necesidades de los usuarios de sus bibliotecas.

Además, debería darse una interacción muy estrecha entre los bibliotecarios y otros profesionales en el área de la biblioteca especializada. De dicha interacción depende en gran medida el que se satisfagan las necesidades de información de los usuarios.

Cabe mencionar otros enfoques relacionados con el desempeño de la biblioteca especializada de acuerdo con los métodos y técnicas administrativas que ha de adoptar el administrador o el responsable del funcionamiento de la biblioteca. En efecto, las medidas que se toman en el campo de la administración contribuyen

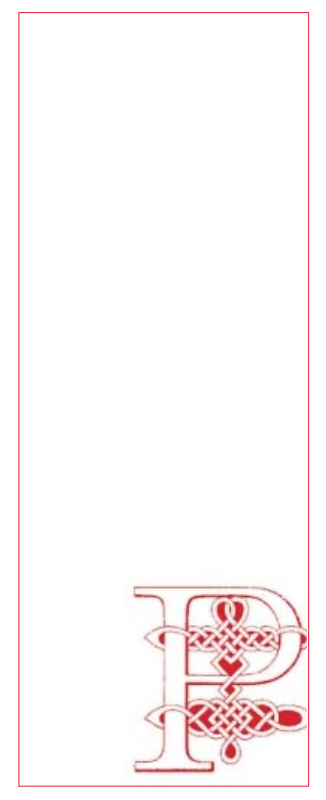

\section{"Debería darse una interacción muy estrecha entre los bibliotecarios y otros profesionales en el área de la biblioteca especializada"}

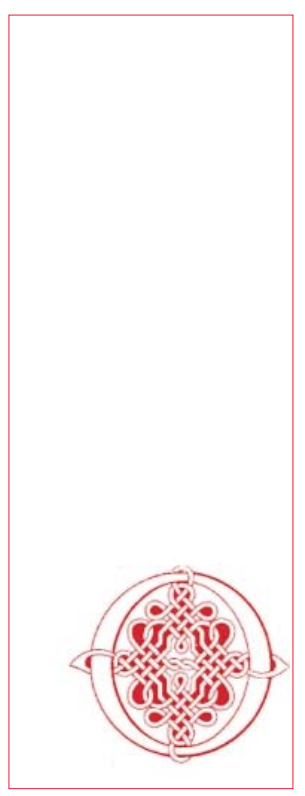

al logro del desempeño eficiente de la biblioteca. No obstante, el propósito del presente artículo de limitará a exponer los beneficios obtenidos del esfuerzo cooperativo, en un intento por descubrir en la cooperación los medios para prestar un mejor servicio a los usuarios de una biblioteca especializada.

No es necesario demostrar el valor de la cooperación, puesto que ya existen numerosos estudios que abordan esta cuestión, además del testimonio ofrecido por las experiencias de múltiples proyectos cooperativos. Es obvio que el éxito siempre depende de la participación de todos los implicados y de su interés en el proyecto. Cuando estén presentes esa conciencia y ese sentido de responsabilidad, tendrá sin duda mejores resultados la unión de esfuerzos.

La cooperación puede empezar dentro de los confines mismos de la biblioteca, y puede extenderse a otras bibliotecas de la misma área, sea en el país o ente varios países como por ejemplo entre los países de América Latina y el Caribe, con lo que se alcanza el nivel internacional.

De este modo, los organismos internaciones interesados en el desarrollo de esta área geográfica han contribuido en gran medida en la coordinación de los esfuerzos de cooperación que se han realizado en el ámbito de la biblioteconomía. Como ejemplo de dichos esfuerzos se encuentran los "Avances de la bibliotecología en el Tercer Mundo” (ALP), realizado por la Federación Internacional de Asociaciones Bibliotecarias (IFLA), los límites geográficos van más allá de América Latina y el Caribe, así como el "Programa regional para el fortalecimiento del sistema y las redes nacionales de información para América Latina y el Caribe" (INFOLAC) realizadas por la UNESCO.

Los proyectos y los programas de cooperación realizados internacionalmente contribuyen, en último término, al desarrollo de la bibliotecología en su conjunto. Es evidente que, en este capo como en cualquier otro, habrá naciones que tienen un mayor desarrollo que otras. Podemos estar seguros de que los programas y proyectos internacionales toman en consideración estos factores durante la etapa de planeación. 
En los ejemplos arriba mencionados, el ALP de la IFLA y la INFOLAC de la UNESCO, ambos programas tienen como objetivo la educación y capacitación de los recursos humanos y una mayor promoción y apoyo para brindar los servicios de información, aunque cada uno de dichos programas tiene sus propios objetivos, metas y proyectos.

En realidad, el desarrollo de estos programas cooperativos se topa con obstáculos para el establecimiento de una política informativa en los países participantes en los programas. En la mayoría de estos países no hay una política nacional al respecto establecida sistemáticamente.

Por esta razón no es fácil llevar estos programas a feliz término, debido al gran número de dificultades que deben afrontarse.

\section{EL PAPEL DE LAS ASOCIACIONES DE BIBLIOTECARIOS EN ESTE CONTEXTO}

Si los bibliotecarios desean mayor reconocimiento y apoyo de los gobiernos, deberán luchar porque el funcionamiento de las bibliotecas sea cada vez más efectivo, ampliando y mejorando la imagen del sector de la información. Sólo de esta forma podrán ganarse y conservar el respeto de la sociedad y, por lo tanto, recibir el mencionado apoyo gubernamental.

Desde esta perspectiva, es importante considerar el papel que desempeñan las asociaciones profesionales. En apoyo de lo anterior Ferguson ${ }^{4}$ señala que las asociaciones formales efectivas son esenciales para cualquier profesión ya que "facilitan la elaboración de planes y la búsqueda activa de programas diseñados para mejorar el bienestar y la condición de sus miembros, promueven el bienestar y la condición de sus miembros, promueven el avance de los proyectos y actividades y la creación de instituciones de apoyo en sus áreas de especialización, refuerzan la filosofía de la profesión y brindan códigos de conducta para sus miembros, ofrecen un frente unificado ante el mundo exterior y garantizan el crecimiento continuo de la profesión al reglamentar la capacitación y atraer miembros de alto nivel gremio". En su opinión, "el avance de la bibliotecología en los países en desarrollo está directamente relacionado con el trabajo de las asociaciones de los bibliotecarios".

Existen, desde luego, algunas barreras. Paez-Urdaneta, apud Ferguson, resume los problemas que viven muchas asociaciones de bibliotecarios en los países en desarrollo:

"Las asociaciones profesionales inexistentes o que sólo existen nominalmente; la automarginación de las actividades gubernamentales en el sector de la información; la automarginación de los programas de capacitación en dicho sector; el bajo nivel de integración nacional, regional o internacional; la escasa o nula participación el las labores de investigación y publicación; el aislamiento respecto de otros profesionales que acuden al sector; la ineficiencia para promover la profesión y mejorar las condiciones económicas y laborales de la fuerza de trabajo del sector informativo; la ineficiencia para promover el concepto de información para el desarrollo, los hábitos de lectura, el acopio o conservación de los documentos nacionales, la modernización de los servicios y el uso de la tecnología informativa."

Si bien Ferguson cree que las asociaciones de bibliotecarios regionales e internacionales han influido sobre la bibliotecología y la creación de asociaciones de bibliotecarios en los países en desarrollo, advierte que "su éxito depende en gran medida de la presencia de las asociaciones nacionales de bibliotecarios, las cualesbrindan el apoyo debido para la instrumentación de programas concebidos en los niveles regional o internacional". ${ }^{4}$

Respecto a las bibliotecas especializadas en un campo específico, se cree que las asociaciones de bibliotecarios de América Latina y el Caribe pueden formular actividades cooperativas de nivel internacional que contribuyan en forma importante al funcionamiento efectivo de las bibliotecas especializadas cuyo fin es satisfacer las necesidades de información de los usuarios.

No obstante, más que una discusión sobre los niveles regional, nacional o internacional de las actividades, hace falta considerar los elementos humanos que entran en juego. Lo que contribuirá con mayor eficacia al éxito en el futuro será el grado de compromiso o interés que muestren los profesionales en las responsabilidades que asuman.

\section{UNA EXPERIENCIA BRASILEÑA}

Para ilustrar lo anterior, se hizo un análisis de una experiencia brasileña en el campo de las Ciencias de la Tierra y la Tecnología Mineral. El análisis de los aspectos positivos y negativos de la experiencia constituyó la base para la sugerencia que se presenta al final de este artículo. Se mencionan también las posibilidades de cooperación entre las bibliotecas de América Latina y el Caribe, como forma alternativa para mejorar el servicio a los usuarios de las bibliotecas especializadas, con el fin de satisfacer mejor las necesidades de información de sus usuarios.

En Brasil existe un gran número de Comisiones Permanentes que tienen lazos con la Federación Brasileña de Asociaciones de Bibliotecarios (FEBAB), las cuales se mantienen activas en todo el país en el contexto de sus campos específicos como, por ejemplo, la Comisión Brasileña para la Documentación Biomédica, la Comisión Brasileña para la Documentación Agrícola, y la Comisión Brasileña para la Documentación Tecnológica, por mencionar sólo algunas.

Estas comisiones forman parte del Consejo Deliberativo de la FEBAB. El objeto de las comisiones es reunir a los bibliotecarios que trabajan en el campo específico de la comisión, a fin de lograr, mediante el esfuerzo y la interacción conjunta con otros profesionales del campo específico, una mejoría al identificar y proporcionar la información necesaria al sector, evitando la duplicación de esfuerzos y con un uso más racional de los recursos humanos, materiales y financieros. Las comisiones deben promover y mantener el intercambio de información entre los grupos de bibliotecarios en los diferentes estados brasileños, a través de las asociaciones de bibliotecarios de cada localidad. Igualmente deben pro- 
mover las organizaciones de bibliotecarios en su campo y fomentar la cooperación entre ellos. Con este fin, deben proponer proyectos y programas para estudios que habrán de realizarse en forma conjunta, difundir noticias y programas de interés para el sector, apoyar la publicación de documentos de interés, mantener un archivo de bibliotecas e intensificar las relaciones con los usuarios del sector. En última instancia, contribuyen de modo importante al mejoramiento de los bibliotecarios, siempre y cuando actúen efectivamente.

El campo que abracaba la Comisión Brasileña para la Documentación Tecnológica era muy amplio. En 1982, los bibliotecarios del campo de las Ciencias de la Tierra y la Tecnología Mineral, interesados en fomentar una más estrecha cooperación entre ellos mismos, empezaron a impulsar los intercambios y a elaborar proyectos de cooperación. El experimento tuvo mucho éxito. Se dieron cuenta de que el número de bibliotecas sobre geología era importante a nivel nacional y justificaba la creación de una comisión específica para este campo. La idea fue aprobada por la Asamblea General de FEBAB, cuando los grupos de bibliotecarios geológicos de Río de Janeiro, São Paulo, Minas Gerais, Bahía, el Distrito Federal, Río Grande do Sul, Paraná y Para se reunieron para formar la Comisión Brasileña para la Documentación en Ciencias de la Tierra y Tecnología Mineral (CBDG).

La CBDG emprendió varios proyectos: la organización de un archivo de las bibliotecas brasileñas sobre Ciencias de la Tierra y Tecnología Mineral, la publicación del Directorio de bibliotecas y documentación, (...) la promoción de conferencias, seminarios, páneles y discusión y cursos según áreas de interés. Preparó una forma para llevar el registro de las colecciones de mapas brasileños y de las colecciones fotográficas.

Fueron evidentes los beneficios derivados del trabajo del la CBDG para ampliar el intercambio de actividades entre los bibliotecarios del sector: mayor conocimiento de la infraestructura de la información sobre Ciencias de la Tierra y Tecnología Mineral, mayor facilidad para el intercambio de información, desarrollo del sector de la información de estos campos, interacción con la comunidad interesada en el campo, mayores oportunidades de identificar las necesidades del usuario mejores condiciones para dar solución a los problemas que afectan la información proporcionada por el sector y utilización máxima de los recursos disponibles.

Las actividades de la comisión avanzaron de manera satisfactoria. En 1987 se celebró un panel sobre información de Ciencias de la Tierra y Tecnología Mineral durante el XXXV Congreso Brasileño sobre Geología, en la ciudad de Belem, Para. El evento fue un éxito y contribuyó a estrechar los lazos entre bibliotecarios y los profesionistas del campo de las Ciencias de la Tierra y la Tecnología Mineral. Por último, aun sin una investigación muy elaborada sobre el tema que brinde una base a este análisis superficial de los puntos positivos y negativos de las actividades de la CBDG, el testimonio de los bibliotecarios y geólogos que colaboraron con la Comisión confirma la validez de este tipo de actividades.

En el momento actual, no son optimistas as perspectivas para las actividades de la Comisión, como tampoco lo son para las actividades en el campo de la minería en su conjunto. Si bien la CBDG ha suspendido sus actividades, podemos decir que durante el periodo de mayor actividad de la Comisión dio gran estímulo al sector de la información en ciencias de la Tierra y Tecnología Mineral.

Por último, aun sin una investigación muy elaborada sobre el tema que brinde una base a este análisis superficial de los puntos posesivos y negativos de las actividades de la CBDG, el testimonio de los bibliotecarios y geólogos que colaboraron con la Comisión confirma la validez de este tipo de actividades.

Desde luego, hay que tomar en cuenta elementos fundamentales como son la unión, la cooperación y la participación, así como la importante labor de los dirigentes bibliotecarios. No es posible prescindir de estos elementos esenciales para la ejecución exitosa de las tareas inherentes al movimiento de las asociaciones. En el sector de la información nadie puede

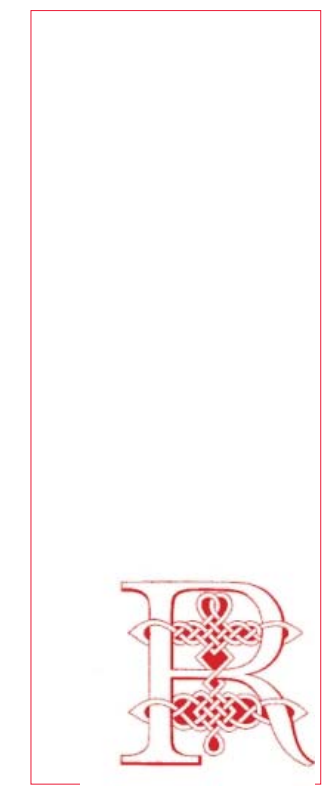

\section{"Corresponde a los bibliotecarios tomar la iniciativa y encontrar la ocasión indicada para salvaguardar su propio espacio profesional."}


pensar en una validación profesional de los bibliotecarios si estos elementos no se hallan presentes en los profesionistas incluidos en esta categoría.

\section{UNA SUGERENCIA PARA EL FUTURO}

Los tiempos actuales ofrecen un reto a los bibliotecarios del futuro. No importa cuáles sean las dificultades qué habrá que enfrentar. Si existen, habrá que vencerlas. Aun cuando la actual situación social y económica de América Latina y el Caribe no sea la idónea, es necesario, ahora más que nunca, unir esfuerzos para tener éxito, venciendo los obstáculos. Vencer la inercia es siempre un buen principio. Una solución podría consistir en buscar el apoyo de los organismos internacionales dedicados a la integración social y económica de América Latina y el Caribe.

Las bibliotecas especializadas en América Latina y el Caribe deberían intentar saber más acerca de los actuales programas de cooperación, a fin de buscar nuevas posibilidades de integración. Si existe interés político nacional por esa integración, las bibliotecas especializadas no podrán ignorar el hecho Corresponde a los bibliotecarios tomar la iniciativa y encontrar la ocasión indicada para salvaguardar su propio espacio profesional.

Una de las formas en que puede alcanzarse este objetivo es con trabajaos como el realizado por las Comisiones Permanentes, en conjunción con la FEBAB en Brasil.

La experiencia descrita anteriormente se limitó a los campos de las Ciencias de la Tierra y la Tecnología Mineral. En otros campos, mientras exista un número importante de bibliotecas para justificar una Comisión a nivel nacional, el trabajo resultará efectivo. Obviamente, será efectivo mientras la unidad, la cooperación, la participación y la organización sigan estando presentes en cada una de las tareas que emprendan.

Si las bibliotecas especializadas de América Latina y el Caribe están realmente interesadas en satisfacer las necesidades de información de sus usuarios, los bibliotecarios deberán comprometerse en el perfeccionamiento constante del sector de la información. No basta pensar en métodos y técnicas. Antes de cualquier cosa, será necesario considerar los recursos humanos, Por ello, si los bibliotecarios son los responsables del sector de la información, les corresponde buscar la mejor manera de satisfacer las necesidades de información de los usuarios.

El trabajo realizado por las Comisiones Permanentes Brasileñas se ofreció como solución alternativa. El trabajo de estas comisiones se basa en la cooperación y los intercambios entre los bibliotecarios y otros profesionistas en el campo específico para el desarrollo de sus actividades.

Otros países de América Latina y el Caribe pueden investigar la posibilidad de esta alternativa de trabajo que se propone. La iniciativa podría representar un paso adelante en el sector de la información en toda América Latina y el Caribe, dando como resultado un avance a nivel internacional en el campo de la bibliotecología.

Ésta es sólo una propuesta, pero pueden surgir otras. Lo indispensable, empero, es buscar siempre alternativas y formas de validar a las bibliotecas y a los bibliotecarios especializados. Sean cuales fueren las formas que adopten estas alternativas y medios, no podrán ignorar la satisfacción de las necesidades de información de los usuarios, puesto que en brindar servicio a estos en América Latina y el Caribe encuentran su razón de ser.

\section{REFERENCIAS BIBLIOGRÁFICAS}

1. Statistical Yearbook for Latin America and the Caribbean; edición de 1990. Santiago de Chile, Comisión Económica para América Latina y el Caribe, 1991, 782 p.

2. Comisión Económica para América Latina y el Caribe, Economic Overview of Latin America (Panorama económico de América Latina), 1991, 84 p., p. 9.

3. ZAMORA, Rosa María Fernández de, "Library Resources in Latin America: a general panorama" (Recursos bibliotecarios en América Latina: un panorama general), IFLA Journal, v. 17, n.1, pp.45-53, 1991

4. FERGUSON, Stephney, "Library Association and the advancement of librarianship in developing countries" (Asociación de Bibliotecas y el desarrollo de la Bibliotecología en los países en desarro1lo). IFLA Journal, v. 17, n.1 pp. 63-71, 1991. 